PROCEEDINGS OF THE

AMERICAN MATHEMATICAL SOCIETY

Volume 131, Number 6, Pages 1847-1855

S 0002-9939(02)06871-5

Article electronically published on November 6, 2002

\title{
GLOBAL EXISTENCE FOR THE CRITICAL GENERALIZED KDV EQUATION
}

\author{
G. FONSECA, F. LINARES, AND G. PONCE
}

(Communicated by David S. Tartakoff)

\begin{abstract}
We discuss results regarding global existence of solutions for the critical generalized Korteweg-de Vries equation,

$$
u_{t}+u_{x x x}+u^{4} u_{x}=0, \quad x, t \in \mathbb{R} .
$$

The theory established shows the existence of global solutions in Sobolev spaces with order below the one given by the energy space $H^{1}(\mathbb{R})$, i.e. solutions corresponding to data $u_{0} \in H^{s}(\mathbb{R}), s>3 / 4$, with $\left\|u_{0}\right\|_{L^{2}}<\|Q\|_{L^{2}}$, where $Q$ is the solitary wave solution of the equation.
\end{abstract}

\section{INTRODUCTION}

Consider the initial value problem

$$
\left\{\begin{array}{l}
u_{t}+u^{4} u_{x}+u_{x x x}=0, \quad x \in \mathbb{R}, \quad t \in \mathbb{R}, \\
u(x, 0)=u_{0}(x) .
\end{array}\right.
$$

We study the global existence of solutions for data in $H^{s}(\mathbb{R}), s \in(0,1)$.

In [8] the local (subcritical) well-posedness of IVP (1.1) was shown. For data in $H^{s}(\mathbb{R}), s>0$, i.e. for any $u_{0} \in H^{s}(\mathbb{R})$, there exist $T\left(\left\|u_{0}\right\|_{s}, s\right)>0$ and a unique solution $u$ of (1.1) in $C\left([0, T]: H^{s}(\mathbb{R})\right)$. It was also proved that for any data $u_{0} \in$ $L^{2}(\mathbb{R})$ there exist $T\left(u_{0}\right)>0$ and a unique strong solution $u$ in $C\left([0, T]: L^{2}(\mathbb{R})\right)$. Moreover, the solution is global for small data.

The latter follows from the local theory in [8] and the fact that $L^{2}$ is a critical space (see the argument below).

Notice that scaling also works for complex-valued functions where the conservation of $\|u(t)\|_{L^{2}}$ does not hold. More precisely, if $u$ solves (1.1), then, for $\lambda>0$, so does

$$
u_{\lambda}(x, t)=\lambda^{1 / 2} u\left(\lambda x, \lambda^{3} t\right),
$$

with data $u_{\lambda}(x, 0)=\lambda^{1 / 2} u(\lambda x, 0)$. It follows that

$$
\left\|u_{\lambda}(\cdot, 0)\right\|_{\dot{H}^{s}}=\lambda^{s}\|u(\cdot, 0)\|_{\dot{H}^{s}} .
$$

Received by the editors January 30, 2002.

2000 Mathematics Subject Classification. Primary 35Q53.

The first author was partially supported by DIB-Universidad Nacional de Colombia.

The second author was partially supported by CNP-q Brazil.

The third author was partially supported by an NSF grant. 
This suggests that the optimal Sobolev index $s$ is $s=0$. Indeed, this result was established in [1]. Hence, the results in 8] imply that complex solutions corresponding to data $u_{0} \in L^{2}(\mathbb{R})$ with

$$
\left\|u_{0}\right\|_{L^{2}} \leq \epsilon_{0}
$$

are global in time.

On the other hand, Weinstein [14] showed the following Gagliardo-Nirenberg estimate for $v \in H^{1}(\mathbb{R})$ and $Q(x)=\left\{3 c \operatorname{sech}^{2}(2 \sqrt{c} x)\right\}^{1 / 4}$, the solitary wave solution of (1.1):

$$
\frac{1}{6} \int v^{6} \leq \frac{1}{2}\left(\frac{\int v^{2}}{\int Q^{2}}\right)^{2} \int v_{x}^{2}
$$

This estimate combined with the conservation laws for real solutions of (1.1),

$$
\int u^{2}(t)=\int u_{0}^{2}
$$

and

$$
\frac{1}{2} \int u_{x}^{2}(t)-\frac{1}{6} \int u^{6}(t)=\frac{1}{2} \int u_{0}^{\prime 2}-\frac{1}{6} \int u_{0}^{6},
$$

gives an a priori estimate in $H^{1}(\mathbb{R})$ provided $\left\|u_{0}\right\|_{L^{2}}<\|Q\|_{L^{2}}$ and therefore the global existence of solutions in $H^{1}(\mathbb{R})$ for initial data satisfying that condition. From this it is clear that $\epsilon_{0}<\|Q\|_{L^{2}}$. Thus if we want to show global existence of solutions in $H^{s}(\mathbb{R}), s \in(0,1)$, we must assume that the initial data satisfy $\epsilon_{0}<\left\|u_{0}\right\|_{L^{2}}<\|Q\|_{L^{2}}$.

Several interesting results have been lately obtained for solutions of IVP (1.1). Merle [12] proved the existence of real-valued solutions of $(1.1)$ in $H^{1}(\mathbb{R})$ corresponding to data $u_{0} \in H^{1}$ satisfying $\left\|u_{0}\right\|_{L^{2}}>\|Q\|_{L^{2}}$ that blow up. There are also various results concerning instability of solitary wave solutions as well as the structure of the blow-up formation obtained by Martel and Merle [10], 11].

In the last few years there have been a great deal of results regarding global existence of solutions for several nonlinear dispersive equations below the energy space. The first result in this direction was obtained by Bourgain [2 for the twodimensional Schrödinger equation. His method was used to obtain such kind of results, for instance, for the $\mathrm{mKdV}$ equation [5] and the $\mathrm{KdV}$ equation [3]; see also 7], [13. Recently, a new variant of Bourgain's method was introduced to obtain sharp global results for the KdV and mKdV equations; see [4]. In [6] for the gKdV-3 equation a sharp local result was obtained which implies $L^{2}$ global well-posedness.

Our main result in this paper reads as follows:

Theorem 1.1. Let $s>3 / 4$. Then for any initial data $u_{0} \in H^{s}(\mathbb{R})$ satisfying $\epsilon_{0}<\left\|u_{0}\right\|_{L^{2}}<\|Q\|_{L^{2}}$, see (1.2), the unique solution of the IVP (1.1) given by the local theory (see Theorem 2.1) extends to any time interval $[0, T]$.

The idea of the proof is similar the one we developed in [5]. That is a combination of sharp smoothing effects present in solutions of the linear problem associated to (1.1) and the iteration process introduced by Bourgain. There are two new elements that can bring some extra difficulty. First, we need to be careful controlling the $L^{2}$ norm of the initial data. We must be in the conditions of the global well-posedness in $H^{1}(\mathbb{R})$. This situation is similar to the one for the derivative Schrödinger equation 
treated by Takaoka in [9. Second, in the local well-posedness result, the time of existence depends on the Sobolev space $H^{s}$ (see below).

We organized this paper as follows: In section 2, we give the statement of the local result obtained in [8] and the main estimates used in the subsequent sections. To prove Theorem 1.1 we need to study two auxiliary IVP's and the properties of their solutions; this will be done in section 3. Finally, the proof of Theorem 1.1 is given in section 4 .

\section{Preliminary Results}

We begin this section with the local sharp result obtained in 8 .

Theorem $2.1([8])$. Let $s>0$. Then for any $u_{0} \in H^{s}(\mathbb{R})$ there exist $T=$ $T\left(\left\|u_{0}\right\|_{s, 2}\right)$ (with $T(\rho, s) \rightarrow \infty$ as $\rho \rightarrow 0$ ) and a unique strong solution $u(\cdot)$ of the IVP (1.1) satisfying

$$
\begin{gathered}
u \in C\left([-T, T]: H^{s}(\mathbb{R})\right), \\
\|u\|_{L_{x}^{5} L_{T}^{10}}+\left\|D_{x}^{s} u\right\|_{L_{x}^{5} L_{T}^{10}}+\left\|D_{t}^{s / 3} u\right\|_{L_{x}^{5} L_{T}^{10}}<\infty
\end{gathered}
$$

and

$$
\left\|\partial_{x} u\right\|_{L_{x}^{\infty} L_{T}^{2}}+\left\|D_{x}^{s} \partial_{x} u\right\|_{L_{x}^{\infty} L_{T}^{2}}+\left\|D_{t}^{s / 3} \partial_{x} u\right\|_{L_{x}^{\infty} L_{T}^{2}}<\infty .
$$

Given $T^{\prime} \in(0, T)$ there exists a neighborhood $V$ of $u_{0}$ in $H^{s}(\mathbb{R})$ such that the map $\tilde{u}_{0} \rightarrow \tilde{u}(t)$ from $V$ into the class defined by (2.1), (2.2) and (2.3) with $T^{\prime}$ instead of $T$ is Lipschitz.

Remark 2.2. From the proof of Theorem 2.1 it follows that the time of existence $T$ satisfies

$$
T \simeq c\left\|u_{0}\right\|_{s}^{-3 / s} .
$$

This can also be deduced from the scaling argument.

The proof of Theorem 2.1 uses as main tools

(1) The smoothing effect of Kato's type associated to the linear problem

$$
\left\{\begin{array}{l}
\partial_{t} u+\partial_{x}^{3} u=0 \\
u(x, 0)=u_{0}(x) .
\end{array}\right.
$$

More precisely, let $U(t)$ be the linear group describing the solution of (2.5), then

$$
\left(\int_{-\infty}^{\infty}\left|\partial_{x} U(t) u_{0}(x)\right|^{2} d t\right)^{1 / 2}=\left\|u_{0}\right\|, \quad \text { for any } x \in \mathbb{R} .
$$

(2) Its dual version, that is,

$$
\left\|\partial_{x} \int_{-\infty}^{\infty} U(-\tau) g(x, \tau) d \tau\right\| \leq c\|g\|_{L_{x}^{1} L_{t}^{2}} .
$$

(3) The double smoothing effect, solutions of the non-homogeneous linear equation

$$
\left\{\begin{array}{l}
\partial_{t} u+\partial_{x}^{3} u=f \\
u(x, 0)=0
\end{array}\right.
$$


satisfy the estimate

$$
\left\|\partial_{x}^{2} u\right\|_{L_{x}^{\infty} L_{T}^{2}} \leq c\|f\|_{L_{x}^{1} L_{T}^{2}}
$$

(4) The fractional derivative commutators.

Theorem 2.3. Let $\alpha \in(0,1), \alpha_{1}, \alpha_{2} \in[0, \alpha], \alpha_{1}+\alpha_{2}=\alpha$. Suppose $p, p_{1}$, $p_{2}, q, q_{1}, q_{2} \in(1, \infty)$ with $\frac{1}{p}=\frac{1}{p_{1}}+\frac{1}{p_{2}}$ and $\frac{1}{q}=\frac{1}{q_{1}}+\frac{1}{q_{2}}$.

$$
\text { Then }
$$

$$
\left\|D_{x}^{\alpha}(f g)-f D_{x}^{\alpha} g-g D_{x}^{\alpha} f\right\|_{L_{x}^{p} L_{T}^{q}} \leq c\left\|D_{x}^{\alpha_{1}} f\right\|_{L_{x}^{p_{1}} L_{T}^{q_{1}}}\left\|D_{x}^{\alpha_{2}} g\right\|_{L_{x}^{p_{2}} L_{T}^{q_{2}}} .
$$

Moreover, for $\alpha_{1}=0$ the value $q_{1}=\infty$ is allowed.

(5) The chain rule for fractional derivatives

Theorem 2.4. Let $\alpha \in(0,1)$, and $p, p_{1}, p_{2}, q, q_{2} \in(1, \infty), q_{1} \in(1, \infty]$ such that $\frac{1}{p}=\frac{1}{p_{1}}+\frac{1}{p_{2}}$ and $\frac{1}{q}=\frac{1}{q_{1}}+\frac{1}{q_{2}}$. Then

$$
\left\|D_{x}^{\alpha} F(f)\right\|_{L_{x}^{p} L_{T}^{q}} \leq c\left\|F^{\prime}(f)\right\|_{L_{x}^{p_{1}} L_{T}^{q_{1}}}\left\|D_{x}^{\alpha} f\right\|_{L_{x}^{p_{2}} L_{T}^{q_{2}}} .
$$

(6) Several interpolation theorems.

These tools plus the contraction mapping principle give the result.

\section{LOCAL WELL-POSEDNESS FOR THE AUXILIARY PROBLEMS}

In this section we describe the properties of two auxiliary IVP's we will use in the proof of Theorem 1.1. We first set some notation.

Let $N$ be a sufficiently large positive number to be chosen later.

Define the norm $\|\cdot\|_{s}$ by

$$
\begin{aligned}
\|u\|_{s} & =\|u\|_{L_{T}^{\infty} H^{s}}+\|u\|_{L_{x}^{5} L_{T}^{10}}+\left\|D_{x}^{s} u\right\|_{L_{x}^{5} L_{T}^{10}}+\left\|D_{t}^{s / 3} u\right\|_{L_{x}^{5} L_{T}^{10}} \\
& +\left\|\partial_{x} u\right\|_{L_{x}^{\infty} L_{T}^{2}}+\left\|D_{x}^{s} \partial_{x} u\right\|_{L_{x}^{\infty} L_{T}^{2}}+\left\|D_{t}^{s / 3} \partial_{x} u\right\|_{L_{x}^{\infty} L_{T}^{2}} .
\end{aligned}
$$

Proposition 3.1. Consider the IVP

$$
\left\{\begin{array}{l}
v_{t}+v^{4} v_{x}+v_{x x x}=0, \quad x \in \mathbb{R}, \quad t>0, \\
v(x, 0)=v_{0}(x) \in H^{1}(\mathbb{R}) .
\end{array}\right.
$$

If $v_{0}$ satisfies

$$
\left\|v_{0}\right\|_{L^{2}}<\|Q\|_{L^{2}} \text { and }\left\|v_{0}\right\|_{H^{1}} \simeq N^{1-s},
$$

then for $\Delta T \simeq\left\|v_{0}\right\|_{H^{1}}^{-3} \simeq c N^{-3(1-s)}$, the existence time given by Theorem [2.1.

(1) The solution $v$ of (3.2) satisfies

$$
\sup _{[0, \Delta T]}\|v(t)\|_{H^{1}} \leq c N^{1-s} .
$$

(2) For any $\rho \in(0,1)$, the solution $v$ of (3.2) satisfies

$$
\|v\|_{\rho} \simeq N^{\rho(1-s)} .
$$

Proof. To prove (3.4) we use (1.5) and (1.3). The estimate (3.5) is established by using Theorem 2.1 and $\left\|v_{0}\right\|_{H^{\rho}} \leq c N^{\rho(1-s)}$. 
Proposition 3.2. Let $w_{0} \in H^{s}(\mathbb{R}), s>0$, and let $v$ be the solution of the IVP (3.2). Then there exists a unique solution $w$ of the IVP

$$
\left\{\begin{array}{l}
\partial_{t} w+\partial_{x}^{3} w+\partial_{x}\left(v^{4} w+2 v^{3} w^{2}+2 v^{2} w^{3}+w^{4} v+\frac{1}{5} w^{5}\right)=0 \\
w(x, 0)=w_{0}(x)
\end{array}\right.
$$

defined in the same interval of existence of $v,[0, \Delta T]$, such that

$$
\begin{gathered}
w \in C\left([0, \Delta T]: H^{s}(\mathbb{R})\right), \\
\|w\|_{L_{x}^{5} L_{\Delta T}^{10}}+\left\|D_{x}^{s} w\right\|_{L_{x}^{5} L_{\Delta T}^{10}}+\left\|D_{t}^{s / 3} w\right\|_{L_{x}^{5} L_{\Delta T}^{10}}<\infty,
\end{gathered}
$$

and

$$
\left\|\partial_{x} w\right\|_{L_{x}^{\infty} L_{\Delta T}^{2}}+\left\|D_{x}^{s} \partial_{x} w\right\|_{L_{x}^{\infty} L_{\Delta T}^{2}}+\left\|D_{t}^{s / 3} \partial_{x} w\right\|_{L_{x}^{\infty} L_{\Delta T}^{2}}<\infty .
$$

Proof. The argument is the same as used in [8] to prove Theorem 2.1 For the sake of clearness we will sketch it.

We use the integral equation form of (3.6) $)$, that is,

$$
w(t)=U(t) w_{0}(x)-\int_{0}^{t} U\left(t-t^{\prime}\right) \partial_{x}\left(v^{4} w+2 v^{3} w^{2}+2 v^{2} w^{3}+w^{4} v+\frac{1}{5} w^{5}\right)\left(t^{\prime}\right) d t^{\prime}
$$

Defining

$$
X_{a, T_{\mathrm{o}}}=\left\{w \in C\left(\left[0, T_{\mathrm{o}}\right]: H^{\rho}(\mathbb{R})\right):\|w\|_{\rho} \leq a\right\},
$$

where $\|\cdot\|$ is as in (3.1), and

$$
\begin{aligned}
\Phi(w) & =\Phi_{v, w_{0}}(w) \\
& \equiv U(t) w_{0}(x)-\int_{0}^{t} U\left(t-t^{\prime}\right) \partial_{x}\left(v^{4} w+2 v^{3} w^{2}+2 v^{2} w^{3}+w^{4} v+\frac{1}{5} w^{5}\right)\left(t^{\prime}\right) d t^{\prime} .
\end{aligned}
$$

Next we shall show that $\Phi$ is a contraction. We will work in detail the case $\left\|D_{x}^{\rho} \Phi\right\|_{L^{2}}$. The other estimates follow similar arguments.

Using the dual of the smoothing effect (2.7), the chain and Leibniz rules for fractional derivatives (2.10) and (2.11), we obtain

$$
\begin{aligned}
& \left\|D_{x}^{\rho} \int_{0}^{t} U\left(t-t^{\prime}\right) \partial_{x}\left(v^{4} w\right)\left(t^{\prime}\right) d t^{\prime}\right\|=\left\|\partial_{x} \int_{0}^{t} U\left(t-t^{\prime}\right) D_{x}^{\rho}\left(v^{4} w\right)\left(t^{\prime}\right) d t^{\prime}\right\| \\
& \leq c\left\|D_{x}^{\rho}\left(v^{4} w\right)\right\|_{L_{x}^{1} L_{T_{\mathrm{o}}}^{2}} \\
& \leq c\left\|D_{x}^{\rho}\left(v^{4}\right) w\right\|_{L_{x}^{1} L_{T_{\mathrm{o}}}^{2}}+c\left\|v^{4}\right\|_{L_{x}^{5 / 4} L_{T_{\mathrm{o}}}^{5 / 2}}\left\|D_{x}^{\rho} w\right\|_{L_{x}^{5} L_{T_{\mathrm{o}}}^{10}} \\
& \leq c\left\|D^{\rho}\left(v^{4}\right)\right\|_{L_{x}^{5 / 4} L_{T_{\mathrm{o}}}^{5 / 2}}\|w\|_{L_{x}^{5} L_{T_{\mathrm{o}}}^{10}}+c\|v\|_{L_{x}^{5} L_{T_{\mathrm{o}}}^{10}}^{4}\left\|D_{x}^{\rho} w\right\|_{L_{x}^{5} L_{T_{\mathrm{o}}}^{10}} \\
& \leq c\|v\|_{L_{x}^{5} L_{T_{\mathrm{o}}}^{10}}^{3}\left\|D_{x}^{\rho} v\right\|_{L_{x}^{5} L_{T_{\mathrm{o}}}^{10}}\|w\|_{L_{x}^{5} L_{T_{\mathrm{o}}}^{10}}+c\|v\|_{L_{x}^{5} L_{T_{\mathrm{o}}}^{10}}^{4}\left\|D_{x}^{\rho} w\right\|_{L_{x}^{5} L_{T_{\mathrm{o}}}^{10}} \\
& \leq c T_{\mathrm{o}}^{4 \rho / 3}\left\|D_{t}^{\rho / 3} v\right\|_{L_{x}^{5} L_{T_{\mathrm{o}}}^{10}}^{3}\left\|D_{x}^{\rho} v\right\|_{L_{x}^{5} L_{T_{\mathrm{o}}}^{10}}\left\|D_{t}^{\rho / 3} w\right\|_{L_{x}^{5} L_{T_{\mathrm{o}}}^{10}} \\
& +c T_{\mathrm{o}}^{4 \rho / 3}\left\|D_{t}^{\rho / 3} v\right\|_{L_{x}^{5} L_{T_{\mathrm{o}}}^{10}}^{4}\left\|D_{x}^{\rho} w\right\|_{L_{x}^{5} L_{T_{\mathrm{o}}}^{10}} \\
& \leq c T_{\mathrm{o}}^{4 \rho / 3}\|v\|_{\rho}^{4}\|w\|_{\rho},
\end{aligned}
$$




$$
\begin{aligned}
& \left\|D_{x}^{\rho} \int_{0}^{t} U\left(t-t^{\prime}\right) \partial_{x}\left(v^{3} w^{2}\right)\left(t^{\prime}\right) d t^{\prime}\right\| \leq c\left\|D_{x}^{\rho}\left(v^{3} w^{2}\right)\right\|_{L_{x}^{1} L_{T_{\mathrm{o}}}^{2}} \\
& \quad \leq c\left\|D_{x}^{\rho} v^{3}\right\|_{L_{x}^{5 / 3} L_{T_{\mathrm{o}}}^{10 / 3}}\left\|w^{2}\right\|_{L_{x}^{5 / 2} L_{T_{\mathrm{o}}^{5}}^{5}}+c\left\|v^{3}\right\|_{L_{x}^{5 / 3} L_{T_{\mathrm{o}}}^{10 / 3}}\left\|D_{x}^{\rho} w^{2}\right\|_{L_{x}^{5 / 2} L_{T_{\mathrm{o}}^{5}}^{5}} \\
& \quad \leq c\|v\|_{L_{x}^{5} L_{T_{\mathrm{o}}^{10}}^{10}}^{2}\left\|D_{x}^{\rho} v\right\|_{L_{x}^{5} L_{T_{\mathrm{o}}^{\mathrm{o}}}^{10}}\|w\|_{L_{x}^{5} L_{T_{\mathrm{o}}}^{10}}^{2}+c\|v\|_{L_{x}^{5} L_{T_{\mathrm{o}}^{10}}^{10}}\left\|D_{x}^{\rho} w\right\|_{L_{x}^{5} L_{T_{\mathrm{o}}^{10}}^{10}}\|w\|_{L_{x}^{5} L_{T_{\mathrm{o}}^{\mathrm{o}}}^{10}} \\
& \quad \leq c T_{\mathrm{o}}^{4 \rho / 3}\|v\|_{\rho}^{3}\|w\|_{\rho}^{2}
\end{aligned}
$$

$$
\begin{aligned}
& \left\|D_{x}^{\rho} \int_{0}^{t} U\left(t-t^{\prime}\right) \partial_{x}\left(v^{2} w^{3}\right)\left(t^{\prime}\right) d t^{\prime}\right\| \leq c\left\|D_{x}^{\rho}\left(v^{2} w^{3}\right)\right\|_{L_{x}^{1} L_{T_{\circ}}^{2}} \\
& \leq c\left\|D_{x}^{\rho} v\right\|_{L_{x}^{5} L_{T_{\mathrm{o}}}^{10}}\|v\|_{L_{x}^{5} L_{T_{\mathrm{o}}}^{10}}\|w\|_{L_{x}^{5} L_{T_{\mathrm{o}}}^{10}}^{3}+c\|v\|_{L_{x}^{5} L_{T_{\mathrm{o}}}^{10}}^{2}\left\|D_{x}^{\rho} w\right\|_{L_{x}^{5} L_{T_{\mathrm{o}}}^{10}}\|w\|_{L_{x}^{5} L_{T_{\mathrm{o}}}^{10}}^{2} \\
& \leq c T_{\mathrm{o}}^{4 \rho / 3}\|v\|_{\rho}^{2}\|w\|_{\rho}^{3},
\end{aligned}
$$

$$
\begin{aligned}
& \left\|D_{x}^{\rho} \int_{0}^{t} U\left(t-t^{\prime}\right) \partial_{x}\left(v w^{4}\right)\left(t^{\prime}\right) d t^{\prime}\right\| \leq c\left\|D_{x}^{\rho}\left(w^{4} v\right)\right\|_{L_{x}^{1} L_{T_{\mathrm{o}}}^{2}} \\
& \quad \leq c\left\|D_{x}^{\rho} v\right\|_{L_{x}^{5} L_{T_{\mathrm{o}}}^{10}}\|w\|_{L_{x}^{5} L_{T_{\mathrm{o}}}^{10}}^{4}+c\|v\|_{L_{x}^{5} L_{T_{\mathrm{o}}}^{10}}\left\|D_{x}^{\rho} w\right\|_{L_{x}^{5} L_{T_{\mathrm{o}}}^{10}}\|w\|_{L_{x}^{5} L_{T_{\mathrm{o}}}^{10}}^{3} \\
& \quad \leq c T_{\mathrm{o}}^{4 \rho / 3}\|v\| \rho\|w\|_{\rho}^{4},
\end{aligned}
$$

and finally

$$
\begin{aligned}
\| D_{x}^{\rho} & \int_{0}^{t} U\left(t-t^{\prime}\right) \partial_{x}\left(w^{5}\right)\left(t^{\prime}\right) d t^{\prime}\|\leq c\| D_{x}^{\rho}\left(w^{4} v\right) \|_{L_{x}^{1} L_{T_{\mathrm{o}}}^{2}} \\
& \leq c\left\|D_{x}^{\rho}\left(w^{4}\right)\right\|_{L_{x}^{5 / 4} L_{T_{\mathrm{o}}}^{5 / 2}}\|w\|_{L_{x}^{5} L_{T_{\mathrm{o}}}^{10}}+c\|w\|_{L_{x}^{5} L_{T_{\mathrm{o}}}^{10}}^{4}\left\|D_{x}^{\rho} w\right\|_{L_{x}^{5} L_{T_{\mathrm{o}}}^{10}} \\
& \leq c\|w\|_{L_{x}^{5} L_{T_{\mathrm{o}}}^{10}}^{4}\left\|D_{x}^{\rho} w\right\|_{L_{x}^{5} L_{T_{\mathrm{o}}^{10}}^{10}} \\
& \leq c T_{\mathrm{o}}^{4 \rho / 3}\|w\|_{\rho}^{5}
\end{aligned}
$$

Combining (3.7) and (3.10) -3.14) we have

$$
\begin{aligned}
\left\|D_{x}^{\rho} \Phi(w)\right\| \leq c\left\|D_{x}^{\rho} w_{0}\right\|+ & T_{\mathrm{o}}^{4 \rho / 3}\left\{\|v\|_{\rho}^{4}+\|v\|_{\rho}^{3}\|w\|_{\rho}\right. \\
& \left.+\|v\|_{\rho}^{2}\|w\|_{\rho}^{2}+\|v\|_{\rho}\|w\|_{\rho}^{3}+\|w\|_{\rho}^{4}\right\}\|w\|_{\rho} \\
\leq c\left\|D_{x}^{\rho} w_{0}\right\|+ & T_{\mathrm{o}}^{4 \rho / 3}\left\{\|v\|_{1}^{4 \rho}+\|v\|_{1}^{3 \rho}\|w\|_{\rho}\right. \\
& \left.+\|v\|_{1}^{2 \rho}\|w\|_{\rho}^{2}+\|v\|_{1}^{\rho}\|w\|_{\rho}^{3}+\|w\|_{\rho}^{4}\right\}\|w\|_{\rho} .
\end{aligned}
$$

Similarly we can obtain estimates for the other norms involved in (3.1) to get

$$
\|\Phi(w)\|_{\rho} \leq\left\|w_{0}\right\|_{H^{\rho}}+T_{\mathrm{o}}^{4 \rho / 3} F\left(\|v\|_{1},\|w\|_{\rho}\right)\|w\|_{\rho} .
$$

Choosing $a=\max \left\{\left\|v_{0}\right\|_{1},\left\|w_{0}\right\|_{\rho}\right\}\left(=\left\|v_{0}\right\|_{1}\right)$ and taking $T_{\mathrm{o}}=\Delta T$ we have

$$
c(\Delta T)^{4 \rho / 3} a^{4}<\frac{1}{2}
$$

Thus $\Phi$ is well defined since $F\left(\|v\|_{1},\|w\|_{\rho}\right) \leq c a^{4}$.

The same argument applies to show that $\Phi$ is a contraction. Hence the contraction mapping principle gives the result. 
Next we record an $L^{2}$-norm growth estimate for solutions of the IVP (3.6).

Lemma 3.3. Let $v$ and $w$ be solutions of the IVP (3.2) and (3.6) with data $v_{0}$ in $H^{1}(\mathbb{R})$ and $w_{0}$ in $H^{s}(\mathbb{R})$ satisfying $\left\|v_{0}\right\|_{H^{1}} \simeq N^{1-s}$ and $\left\|w_{0}\right\|_{2} \simeq N^{-s}, 0<s<1$, respectively. Define

$$
\|w\|_{0}=\sup _{[0, \Delta T]}\|w(t)\|_{L^{2}}+\|w\|_{L_{x}^{5} L_{\Delta T}^{10}}+\left\|\partial_{x} w\right\|_{L_{x}^{\infty} L_{\Delta T}^{2}} .
$$

Then

$$
\|w\|_{0} \simeq N^{-s} .
$$

\section{Proof of Theorem 1.1}

Consider the solution of the IVP

$$
\left\{\begin{array}{l}
u_{t}+u^{4} u_{x}+u_{x x x}=0, \quad x \in \mathbb{R}, \quad t \in \mathbb{R} \\
u(x, 0)=u_{0}(x)
\end{array}\right.
$$

For $u_{0} \in H^{s}(\mathbb{R}), 0<s<1$, with $\left\|u_{0}\right\|_{L^{2}}<\|Q\|_{L^{2}}$, we split $u_{0}$ as

$$
u_{0}(x)=v_{0}(x)+w_{0}(x)=\left(\hat{u}_{0} \chi_{|\xi| \leq N}\right)^{\vee}+\left(\hat{u}_{0} \chi_{|\xi|>N}\right)^{\vee} .
$$

$N \gg 1$ will be determined later. Observe that

$$
\left\|v_{0}\right\|_{L^{2}}<\|Q\|_{L^{2}}, \quad\left\|v_{0}\right\|_{H^{1}} \simeq N^{1-s}
$$

and

$$
\left\|w_{0}\right\|_{\rho} \simeq N^{\rho-s}, \quad \text { for } \quad 0<\rho \leq s<1 .
$$

Using Propositions 3.1 and 3.2 we have solutions $v(t) \in H^{1}(\mathbb{R})$ and $w(t) \in H^{\rho}(\mathbb{R})$ such that $u(t)=v(t)+w(t)$, for $t \in[0, \Delta T]$ where $\Delta T \simeq\left\|v_{0}\right\|_{H^{1}}^{-3} \simeq N^{-3(1-s)}$.

Our goal is to extend the time of existence of (4.1) up to any time $T>0$.

To do so we use an iteration scheme that we will describe below.

First notice that from Proposition 3.2 we have

$$
w(t)=U(t) w_{0}(x)+z(t)
$$

where

$$
z(t)=-\int_{0}^{t} U\left(t-t^{\prime}\right) \partial_{x}\left(v^{4} w+2 v^{3} w^{2}+2 v^{2} w^{3}+w^{4} v+\frac{1}{5} w^{5}\right)\left(t^{\prime}\right) d t^{\prime}, \quad t \in[0, \Delta T] .
$$

Hence the solution $u$ of (4.1) can be written as

$$
u(t)=v(t)+U(t) w_{0}+z(t)
$$

for any $t \in[0, \Delta T]$. In the process of iteration we need to check the growth of the solution at each interval of size $\Delta T$.

We observe that at the point $t=\Delta T$

$$
u(\Delta T)=v(\Delta T)+U(\Delta T) w_{0}+z(\Delta T),
$$

and that $U(\Delta T) w_{0}$ stays in $H^{s}(\mathbb{R})$ by group properties. Hence we shall show that for $v(\Delta T)+z(\Delta T)$ the conditions (4.2) hold.

From Proposition 3.1

$$
\|v\|_{L_{t}^{\infty} H^{1}} \leq c N^{(1-s)} .
$$

On the other hand,

$$
\|z\|_{L_{\Delta T}^{\infty} H^{1}} \leq c N^{(1-2 s)} .
$$


In fact, the worst term in $z(t)$ is $\partial_{x}\left(v^{4} w\right)$. Thus we estimate

$$
\left\|\partial_{x} \int_{0}^{\Delta T} U\left(t-t^{\prime}\right) \partial_{x}\left(v^{4} w\right)\left(t^{\prime}\right) d t^{\prime}\right\|_{L^{2}}
$$

and

$$
\left\|\int_{0}^{\Delta T} U\left(t-t^{\prime}\right) \partial_{x}\left(v^{4} w\right)\left(t^{\prime}\right) d t^{\prime}\right\|_{L^{2}} .
$$

The dual version of the smoothing effect (2.7), Hölder's inequality and Sobolev's embedding give

$$
\begin{array}{rl}
\| \partial_{x} \int_{0}^{\Delta T} & U\left(t-t^{\prime}\right) \partial_{x}\left(v^{4} w\right)\left(t^{\prime}\right) d t^{\prime}\left\|_{L^{2}} \leq\right\| v^{4} \partial_{x} w\left\|_{L_{x}^{1} L_{\Delta T}^{2}}+\right\| v^{3} \partial_{x} v w \|_{L_{x}^{1} L_{\Delta T}^{2}} \\
& \leq\|v\|_{L_{x}^{4} L_{\Delta T}^{\infty}}^{4}\left\|\partial_{x} w\right\|_{L_{x}^{\infty} L_{\Delta T}^{2}}+\|v\|_{L_{x}^{5} L_{\Delta T}^{10}}^{3}\left\|\partial_{x} v\right\|_{L_{x}^{5} L_{\Delta T}^{10}}\|w\|_{L_{x}^{5} L_{\Delta T}^{10}} \\
& \leq\|v\|_{L_{x}^{4} L_{\Delta T}^{\infty}}^{4}\left\|\partial_{x} w\right\|_{L_{x}^{\infty} L_{\Delta T}^{2}}+\Delta T\left\|D_{t}^{1 / 3} v\right\|_{L_{x}^{5} L_{\Delta T}^{10}}^{3}\left\|\partial_{x} v\right\|_{L_{x}^{5} L_{\Delta T}^{10}}\|w\|_{L_{x}^{5} L_{\Delta T}^{10}} \\
& \leq c\|v\|_{1 / 4}^{4}\|w\|_{0}+\Delta T\|v\|_{1}^{4}\|w\|_{0} \\
& \leq c\|v\|_{1}\|w\|_{0}+\Delta T\|v\|_{1}^{4}\|w\|_{0} \\
& \leq c N^{(1-s)} N^{-s}+c N^{-3(1-s)} N^{4(1-s)} N^{-s} \\
& \leq c N^{(1-2 s)}
\end{array}
$$

A similar argument as in 4.5 yields

$$
\begin{aligned}
& \| \int_{0}^{\Delta T} U\left(t-t^{\prime}\right) \partial_{x}\left(v^{4} w\right)\left(t^{\prime}\right) d t^{\prime}\left\|_{L^{2}}=\right\| \partial_{x} \int_{0}^{\Delta T} U\left(t-t^{\prime}\right)\left(v^{4} w\right)\left(t^{\prime}\right) d t^{\prime} \|_{L^{2}} \\
& \leq\|v\|_{L_{x}^{5} L_{T}^{10}}^{4}\|w\|_{L_{x}^{5} L_{T}^{10}} \leq(\Delta T)^{4 / 3}\left\|D_{t}^{1 / 3} v\right\|_{L_{x}^{5} L_{T}^{10}}^{4}\|w\|_{L_{x}^{5} L_{T}^{10}} \\
& \leq(\Delta T)^{4 / 3}\|v\|_{1}^{4}\|w\|_{0} \leq c N^{-4(1-s)} N^{4(1-s)} N^{-s} \\
& \leq c N^{-s} .
\end{aligned}
$$

From (4.5) and (4.6) we obtain (4.4).

We also have that

$$
\begin{aligned}
\|v(\Delta T)+z(\Delta T)\|_{L^{2}} & =\left\|u(\Delta T)-U(\Delta T) w_{0}\right\|_{L^{2}} \\
& \leq\left\|u_{0}\right\|_{L^{2}}+\left\|w_{0}\right\|_{L^{2}} \\
& \leq\left\|u_{0}\right\|_{L^{2}}+N^{-s}
\end{aligned}
$$

where we have used the conservation law (1.4) and group properties. So if $N$ is sufficiently large, we will have

$$
\|v(\Delta T)+z(\Delta T)\|_{L^{2}}<\|Q\|_{L^{2}} .
$$

Thus at each step there is a contribution of $N^{(1-2 s)}$ from $\|z\|_{L_{\Delta T}^{\infty} H^{1}}$.

Hence, to reach the time $T$, the needed number of iterations is $\frac{T}{\Delta T}$, so if we want to guarantee that the $H^{1}$-norm grows on the interval $[0, T]$ as $N^{(1-s)}$, that is,

$$
\frac{T}{\Delta T} N^{(1-2 s)} \sim T N^{3(1-s)} N^{(1-2 s)} \leq c N^{(1-s)},
$$

we must choose $N(T) \sim T^{\frac{1}{4 s-3}}$ for $3 / 4<s<1$. 
From (4.7) we also have a contribution at each step from the $L^{2}$-norm of $N^{-s}$. Therefore to reach $T$ we have

$$
T N^{3(1-s)} N^{-s} \leq c
$$

which is satisfied by the $N(T)$ previously chosen. This completes the proof of the theorem.

\section{REFERENCES}

[1] B. Birnir, C. E. Kenig, G. Ponce, N. Svanstedt and L. Vega, On the ill-posedness of the IVP for the generalized Korteweg-de Vries and nonlinear Schrödinger equations, J. London Math. Soc. (2), 53 (1996), 551-559. MR 97d:35233

[2] J. Bourgain, Refinements of Strichartz' inequality and applications to $2 D$-NLS with critical nonlinearity, Internat. Math. Res. Notices, 5 (1998), 253-283. MR 99f:35184

[3] J. Colliander, G. Staffilani and H. Takaoka, Global well-posedness for KdV below $L^{2}$, Math. Res. Lett., 6 (1999), 755-778. MR 2000m:35159

[4] J. Colliander, M. Keel, G. Staffilani, H. Takaoka and T. Tao, Sharp global well-posedness for $K d V$ and modified $K d V$ on $\mathbb{R}$ and $\mathbb{T}$, preprint.

[5] G. Fonseca, F. Linares and G. Ponce, Global well-posedness for the modified Korteweg-de Vries equation, Communications in PDE, 24 (3\&4) (1999), 683-705. MR 2000a:35210

[6] A. Grünrock, A bilinear Airy-estimate with applications to $g K d V$-3, preprint.

[7] C. E. Kenig, G. Ponce and L. Vega, Global well-posedness for semi-linear wave equations, Comm. Partial Differential Equations, 25 (2000), 1741-1752. MR 2001h:35128

[8] C. E. Kenig, G. Ponce and L. Vega, Well-posedness and scattering results for the generalized Korteweg-de Vries equation via the contraction principle, Comm. Pure Appl. Math., 46 (1993), 527-620. MR 94h:35229

[9] H. Takaoka, Global well-posedness for Schrödinger equations with derivatives in a nonlinear term and data in low-order Sobolev spaces, Electronic J. Differential Equations, 42 (2001). MR 2002f:35033

[10] Y. Martel and F. Merle, Instability of solitons for the critical generalized Korteweg-de Vries equation, Geom. Funct. Anal., 11 (2001), 74-123. MR 2002g:35182

[11] Y. Martel and F. Merle, Stability of blow-up profile and lower bounds for blow-up rate for the critical generalized KdV equation, Annals of Math. (2) 155 (2002), 235-280.

[12] F. Merle, Existence of blow-up solutions in the energy space for the critical generalized KdV equation, J. Amer. Math. Soc., 14 (2001), 555-578. MR 2002f:35193

[13] H. Pecher, Global well-posedness below energy space for the 1-dimensional Zakharov system, Internat. Math. Res. Notices, 19 '(2001), 1027-1056. MR 2002j:35036

[14] M. Weinstein, Lyapunov stability of ground states of nonlinear dispersive evolution equations, Comm. Pure Appl. Math., 39 (1986), 51-67. MR 87f:35023

Departamento de Matemáticas, Universidad nacional de Colombia, Bogotá, ColomBIA

E-mail address: gfonseca@matematicas.unal.edu.co

Instituto de Matemática Pura e Aplicada, 22460-320, Rio de Janeiro, Brazil

E-mail address: linares@impa.br

Department of Mathematics, University of California, Santa Barbara, California 93106

E-mail address: ponce@math.ucsb.edu 